\title{
9 Resümee: Modellierungen verkörperten Denkens durch filmisches Metaphorisieren - (mit) VERTIGo wahrnehmen, fühlen und verstehen
}

In ihrer Auffassung von Semiose als einer „zeitlichen Struktur [...], mit der das Wahrnehmen und Denken, die sinnlich-psychische und die kognitive Bewegung des Zuschauens in den Bildraum des Films eingewoben sind“, ${ }^{1}$ verschränkt sich nun die Theorie filmischer Expressivität mit der sprach- und gestenwissenschaftlichen Auffassung von Metapher als einem dynamischen, situativ verorteten und verkörperten Prozess des Denkens und Herstellens von Bedeutung, wie sie eingangs dieses zweiten Teils der Arbeit dargelegt wurde. Denn es ist eine „sich nach und nach entfaltende Metaphorizität “, ${ }^{2}$ der das Vermögen zugesprochen wird, eine zeitliche, rhythmische Oberflächenstrukturierung zu sein, die eine solche Bewusstseinsbewegung präformiert. Hermann Kappelhoff und Cornelia Müller haben diese Verschränkung mit dem Begriff der „Cinematic Metaphor“3 gefasst. Die Rede vom filmischen Metaphorisieren in der vorliegenden Arbeit schließt unmittelbar daran an und stellt zugleich eine zentrale Charakteristik filmischer Metaphern in den Vordergrund: nämlich, dass diese immer in der kontextgebundenen Dimension eines Machens, einer Aktivität zu denken sind. ${ }^{4}$

1 Kappelhoff: And the heart will go on and on, S. 105.

2 Ebd., S. 106, Herv. CS.

3 Müller/Kappelhoff: Cinematic Metaphor. Erstmalig findet sich die Verschränkung ausgearbeitet in Kappelhoff/Müller: Embodied Meaning Construction. Vgl. auch Christina Schmitt/Sarah Greifenstein/Hermann Kappelhoff: Expressive Movement and Metaphoric Meaning Making in AudioVisual Media. In: Body - Language - Communication. An International Handbook on Multimodality in Human Interaction, Vol. 2. Hrsg. v. Cornelia Müller, Alan Cienki, Ellen Fricke, Silva H. Ladewig, David McNeill und Jana Bressem. Berlin/New York: De Gruyter Mouton 2014, S. 2092-2112; Schmitt: Embodied Meaning in Audio-Visuals; Müller/Schmitt: Audio-Visual Metaphors of the Financial Crisis; Hermann Kappelhoff/Sarah Greifenstein: Audiovisual Metaphors. Embodied Meaning and Processes of Fictionalization. In: Embodied Metaphors in Film, Television, and Video Games. Cognitive Approaches. Hrsg. v. Kathrin Fahlenbrach. New York/London: Routledge 2015, S. 183201; Hermann Kappelhoff/Sarah Greifenstein: Metaphorische Interaktion und empathische Verkörperung. Thesen zum filmischen Erfahrungsmodus. In: Empathie im Film. Perspektiven der ästhetischen Theorie, Phänomenologie und Analytischen Philosophie. Hrsg. v. Malte Hagener und Íngrid Vendrell Ferran. Bielefeld: transcript 2016, S. 167-193; Greifenstein/Horst/Scherer/Schmitt/ Kappelhoff/Müller (Hrsg.): Cinematic Metaphor in Perspective; Horst: Meaning-Making and Political Campaign Advertising; Kappelhoff: Kognition und Reflexion; Greifenstein: Tempi der Bewegung Modides Gefühls.

4 So findet sich in der gebrauchsorientierten Metaphernforschung im englischsprachigen Bereich gegenwärtig auch verstärkt die Rede von „doing metaphor“ (vgl. z. B. Thomas Wiben Jensen:

Ә Open Access. () 2020 Christina Schmitt, publiziert von De Gruyter. (C) BY-NC-ND Dieses Werk ist lizenziert unter der Creative Commons Attribution-NonCommercial-NoDerivatives 4.0 Lizenz.

https://doi.org/10.1515/9783110614619-009 
Zum Abschluss von Teil II, der im Zeichen der Entwicklung eines vom Gebrauch ausgehenden Verständnisses von ,Metapher‘ im Kontext audiovisueller Bilder steht und hierfür eine Denkbewegung vom Sprachgebrauch zum FilmeSehen vollzogen hat, führe ich nun meine Analyse von vERTIGo weiter fort. Was für ein Wahrnehmen, Fühlen und Verstehen ereignet sich hier, was für ein filmisches Metaphorisieren?

Noch einmal also: die Szene in Ernie's Restaurant ${ }^{5}$ - diese dialogfreie Szene des erstmaligen Sehens von Madeleine durch Scottie, in der die basale Dimension der cinematic communication (das Hören eines Hörens, das Sehen eines Sehens, das Spüren einer Bewegung) so exponiert ist, weil das Wahrnehmungserleben selbst im Fokus ist.

Für die Szene wurde konstatiert, dass in ihrer Expressivität ein spezifisches Bild des Sehens wahrnehmbar gemacht, in Szene gesetzt wurde. Dies deutete bereits an, was nun noch einmal explizit formuliert sei. Nämlich, dass hier durch die Art und Weise, wie Kamerabewegung, Montage, Sound, Bildkomposition und Schauspiel zusammenwirken, ein filmisches Metaphorisieren modelliert ist. Dass ein Erfahrungsbereich durch die Wahrnehmung eines anderen verstanden wird. Denn es ist ein auf Distanz bedachtes, ungesehenes, indirektes Sehen, ein Sehen des Beobachtens und Beschattens ohne Blickkontakt, das hier durch ein spezifisches gegenteiliges Empfinden wahrgenommen wird: ein Empfinden des InKontakt-Kommens, das sich über eine szenische Komposition von drei Ausdrucksbewegungen entfaltete. Zur Erinnerung: Im Verlauf der Szene transformierte sich eine gedehnte und ungerichtete Wahrnehmungsbewegung in eine sanft soghafte, fokussierte, sich spannungsvoll in ihrer Intensität steigernde Annäherung - die ihren Höhepunkt im Erleben eines flüssigen Miteinander-Verschmelzens in einer sich ergießenden Drehbewegung fand und in einem sanften Voneinander-Lösen ausklang.

Eine Fiktionalisierung, ein narratives Verständnis dieser Szene, ein Figurenerleben - dass sich der ehemalige Polizist Scottie hier ,auf den ersten Blick ${ }^{*}$ verliebt, ja, dass er hier dem Schwindel eines für ihn inszenierten Madeleine-Bildes verfällt - ist durch die Ausdrucksbewegungsbilder und ihre Hervorbringungsweise noch allererst evoziert und durch sie ermöglicht. ${ }^{6}$

Doing Metaphor. An Ecological Perspective on Metaphoricity in Discourse. In: Metaphor. From Embodied Cognition to Discourse. Hrsg. v. Beate Hampe. Cambridge: Cambridge University Press 2017, S. 257-276; Raymond W. Gibbs, Jr.: Metaphor and Human Experience. RaAM Specialized Seminar: Ecological Cognition and Metaphor. University of Southern Denmark, 18.05.2017).

5 Vgl. die Kapitel 8.2 und 8.3 (dort die Ausführungen zur filmischen Expressivität) der vorliegenden Arbeit für die bisherigen verTigo-Analysen.

6 Vgl. zur Fiktionalisierung Kappelhoff: Kognition und Reflexion. 


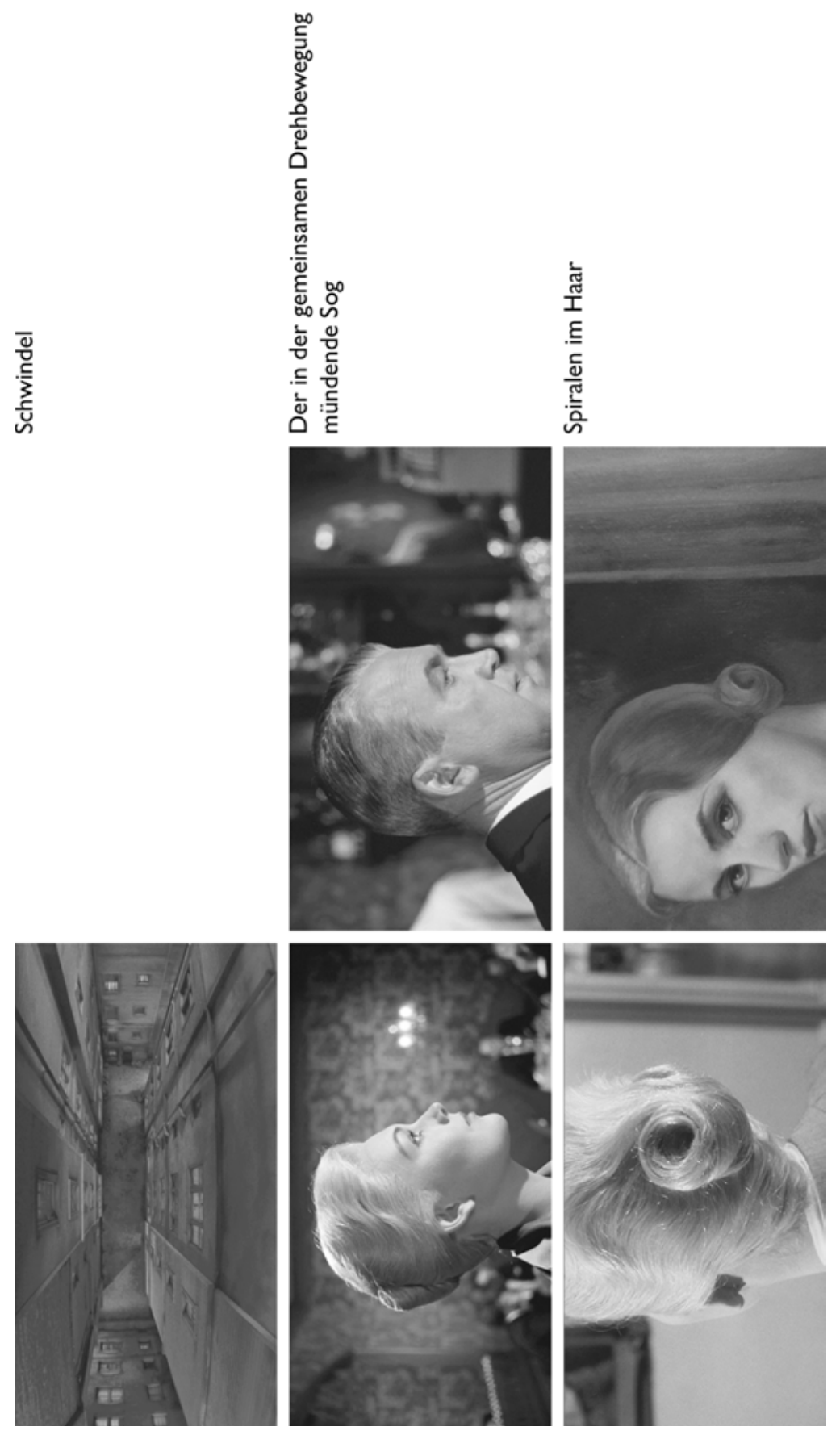




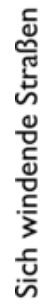

몬

㐫
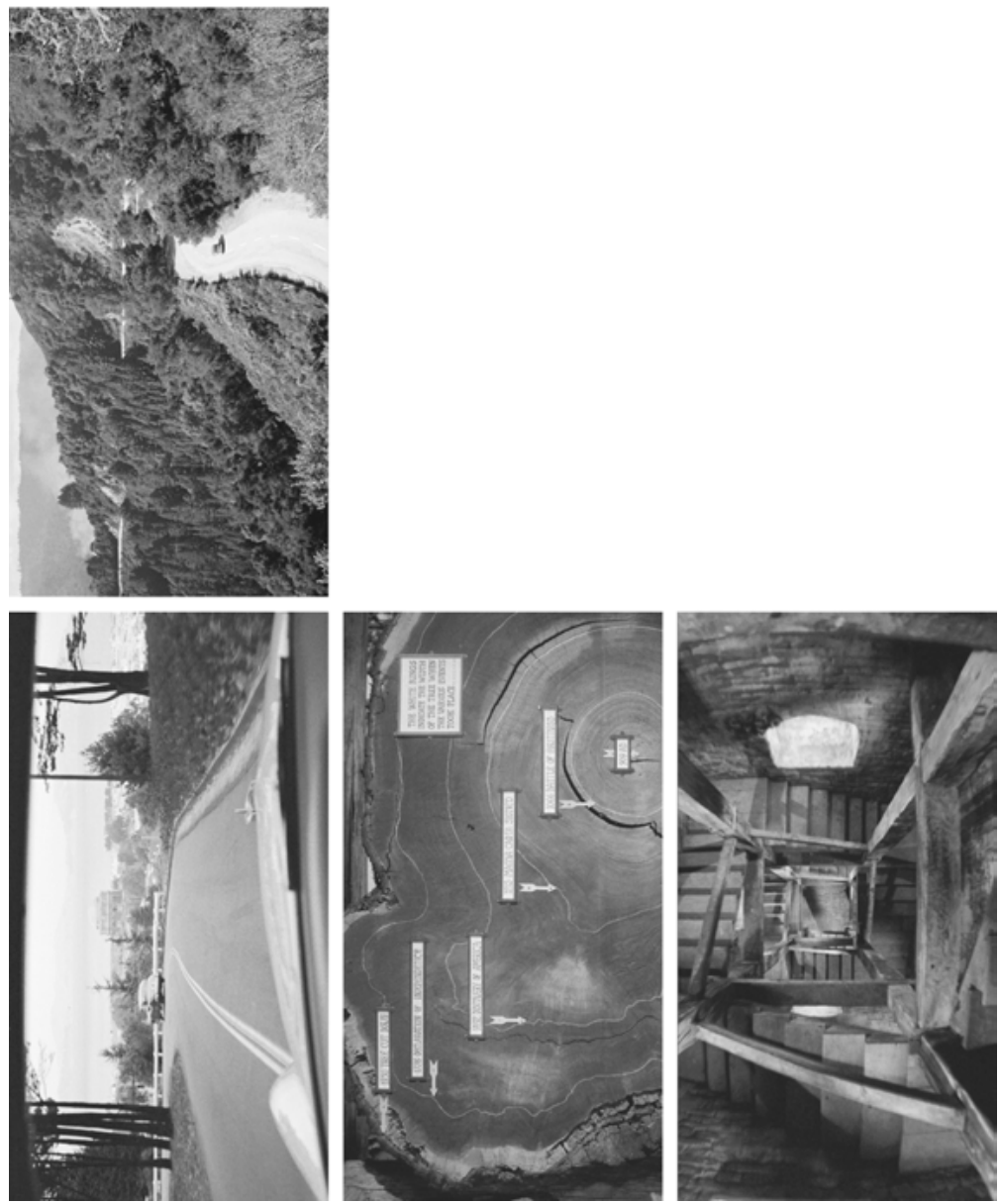

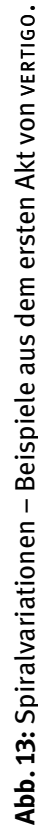


Zugleich ist die Szene über ihre Erfahrungsform in größere Komplexe und Verzweigungen eingebunden. So ist der in einer gemeinsamen Drehbewegung mündende Sog in makroanalytischer Perspektive Teil des dynamischen Relationsgefüges von VERTIGo als Ganzem, das getragen wird von einem Geflecht unterschiedlicher Spiralen: ${ }^{7}$ das Schwindelgefühl im Angesicht des tödlichen Abgrunds, mit dem alles beginnt; das (selbst noch in zahllosen Varianten) gedrehte Haar Madeleines, der todgeweihten Doppelgängerin ihrer Urgroßmutter und Selbstmörderin Carlotta Valdes; Fahrten auf sich windenden Straßen, wie etwa bei einer Verfolgung und der Fahrt Scotties und Madeleines zu einem Wald mit riesigen Bäumen, „always green, ever living“, wo Madeleine in den Jahresringen eines Baumes ihre Geburt wie ihren in der Vergangenheit liegenden Tod markiert (Abb. 13).

Hierüber entfalten und verweben sich ein In-Abgründe-Stürzen und (Ver-) Lieben, ein falling und (falling in) love, Todes- und Liebessehnsucht metaphorisch miteinander. Und in gewisser Weise entfaltet der Vorspann dieses Variationsspiel in konzentrierter Weise gleichsam metonymisch für den gesamten Film (0:00:220:03:13, Abb. 14)*: Ruhig gleitet der Blick in Detail-Schwarzweiß-Aufnahme über ein Frauengesicht; über dem vollen Mund blendet „James Stewart“ ein; der Blick wandert weiter nach oben, bis zu angstvollen Augen, unter denen „Kim Novak“ einblendet; und weiter wandert der Kamerablick, fokussiert ein sich weitendes Auge, und aus dessen Tiefen - das Bild hat sich nun in tiefes Rot gefärbt - steigt „Vertigo" auf und füllt schließlich das gesamte Bildfeld in seiner Breite. Und dann taucht aus diesem Auge eine sich drehende Spirale auf, das Bild wird mit den Credits nun gänzlich grafisch, und das Spiel der Spiral-Variationen nimmt seinen audiovisuellen Lauf. Optisch realisiert sich dies in ganz verschiedenen, sich unterschiedlich schnell bewegenden Spiralen in den prägnanten Farben des Films, die stetig, in gemäßigtem Tempo auftauchen - Rot (das Rot des Verliebens), Grün (das Grün des Phantasma des Begehrens: Madeleine), Gelb (das Gelb der Blumen auf dem Friedhof mit Carlottas Grab), Blau (das Blau des Meeres des ersten Kusses), Violett (das Violett des Nicht-Madeleine-Seins von Judy) - bis schließlich das Auge wiederkommt. Verwoben ist dies mit einer Mollkomposition, deren hohe und schnelle Tonverläufe der Streicher sich in konträre Richtungen winden - gleichsam einer musikalischen Variante von Hitchcocks berühmtem visuellem Vertigo-Effekt des zeitgleichen Einzoomens und Zurückfahrens der Kamera $^{8}$-, verstärkt von Disharmonien durchzogen; hierein brechen immer wieder, langgezogen, impulsiv und heftig, tiefe Hörner und Posaunen ein und bringen dadurch die Höhe der Streicher noch mit hervor.

7 Vgl. auch Deleuze: Das Bewegungs-Bild, S. 39f., 274.

8 Vgl. Schneller: Death and Love, S. 194. 

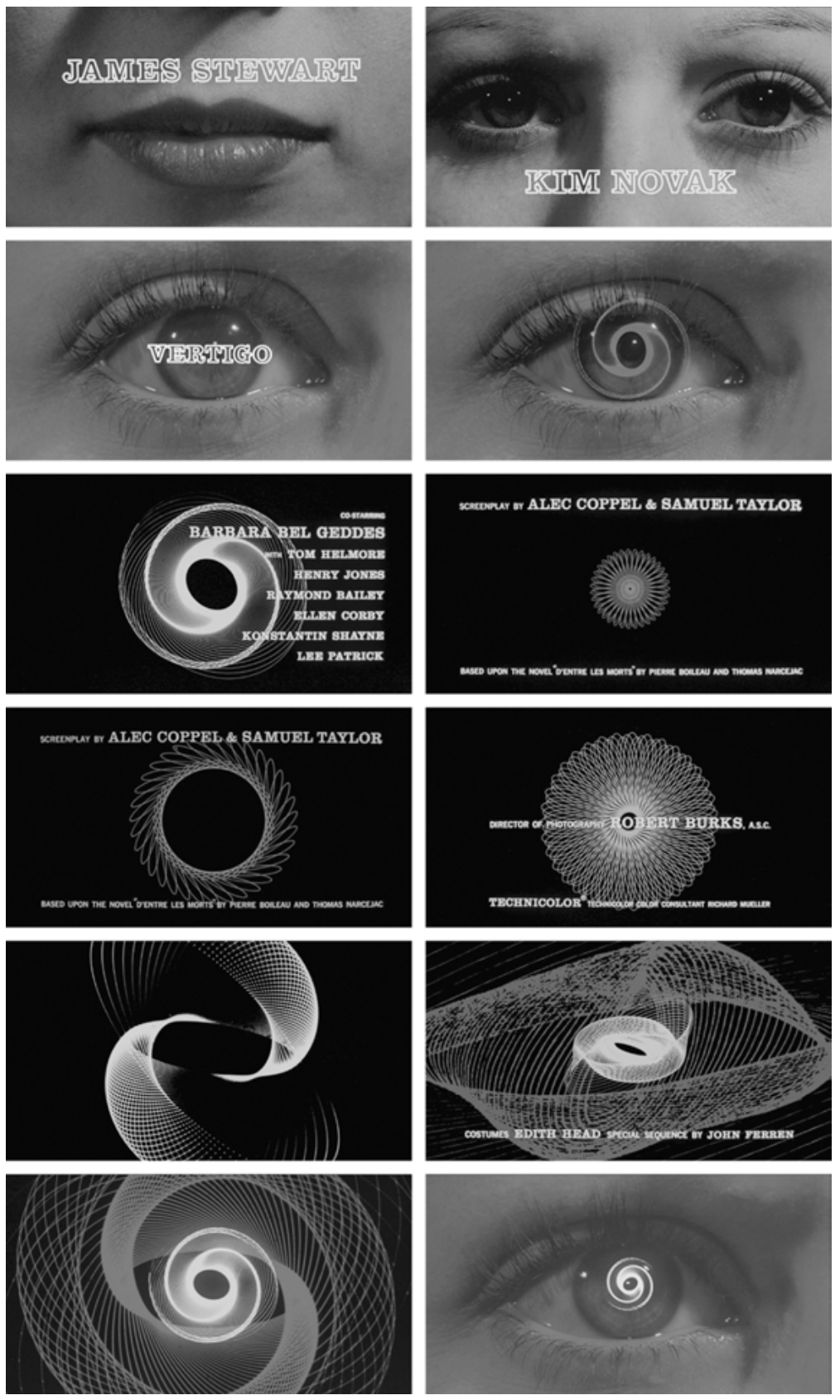

Abb. 14: Der Vorspann als Metonymie des gesamten VERTIGo-Films: Variationsspiel der Spiralen. $\left(\rightarrow\right.$ Abb. $14^{*}$, Farbbogen) 
Über seine Farbigkeit ist der Bildraum des Begehrens, wie er sich in der Restaurant-Szene entfaltet, zugleich Keimzelle eines Metaphorisierens, das Liebe und Tod als wechselseitige Entsprechung, als Komplementärverhältnis entstehen lässt. Einen Parcours von Ausdrucksqualitäten durchlaufend, durchzieht das RotGrüne von nun an den gesamten Film - und entfaltet im Ganzen eine Bewegung vom Rot zum Grün.

So folgt dem Rot des ersten Gewahrwerdens des Grünen, wie es sich in der Restaurantszene ereignet (0:16:10-0:17:45, Abb.10-12)^, alsbald das zwischen lauter gelben Blumen plötzlich auftauchende rote Blumenmeer auf dem grünen Friedhof, zu dem Scottie Madeleine folgt - angespannt-zögernd-unausgewogen/ disharmonisch (0:21:18-0:24:21 / letzte Ausdrucksbewegungseinheit einer szenischen Komposition, Dominanten: Montage und Musik / Abb. 15)^; ...

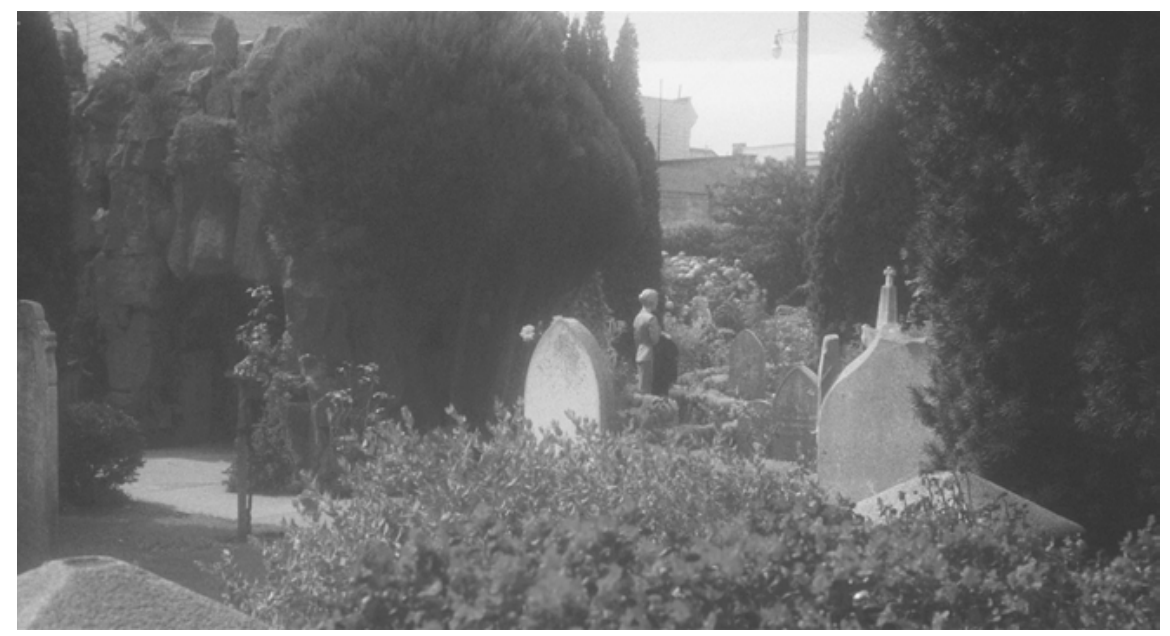

Abb. 15: vertigo. ( $\rightarrow$ Abb. 15*, Farbbogen)

... die Bewegung setzt sich fort in der rot-grünen Bildkomposition der Szene des ersten Gesprächs und der Annäherung von Scottie und Madeleine (nach Madeleines Fall ins grüne Meer und der Rettung durch Scottie) - ein mehrfach sich wiederholendes, relativ moderates und getragenes Wechseln zwischen Entspannung und Anspannung (0:42:04-0:50:52 / gesamte szenische Komposition, Dominanten: Schauspiel und Musik / Abb. 16)^. Auch verbal wird das Rot Madeleines, das Komplementäre, in dieser Szene indirekt hervorgehoben: Mit den Worten „you want this“ drückt Scottie, auf den Madeleines Grün übergegangen ist, der unter der Bettdecke Entkleideten den roten Morgenmantel in die Hand; ... 

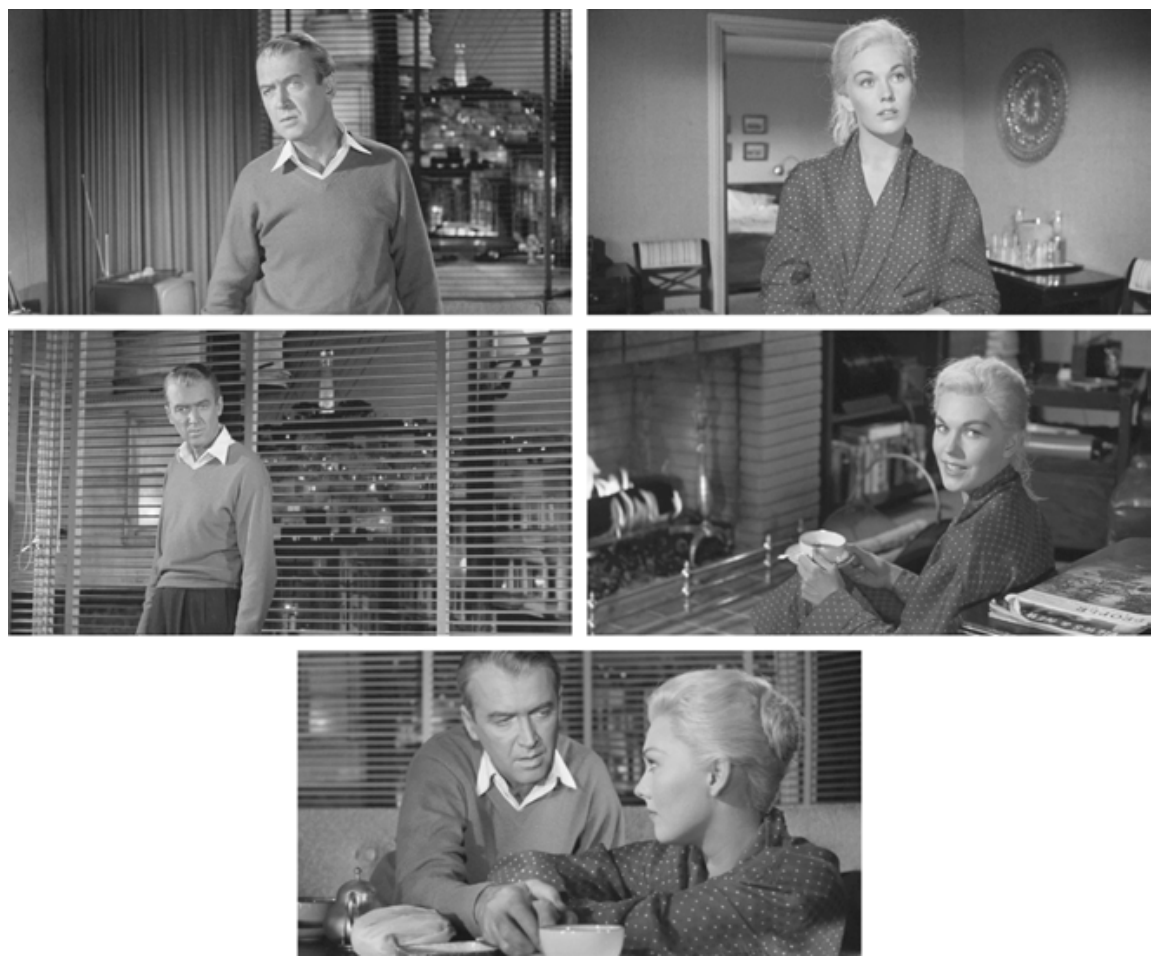

Abb. 16: Vertigo. ( $\rightarrow$ Abb. 16*, Farbbogen)

... sie setzt sich fort im rot-grünen Wald des Dialogs von Scottie und Madeleine über das Immer-Grüne, das Ewig-Lebende, das Wissen um den eigenen zukünftigen und vergangenen Tod - leise-dunkel-gedehnt, disharmonisch, immer wieder an- und abschwellend, starrwerdend (0:55:56-0:59:28 / gesamte szenische Komposition, Dominanten: Bildkomposition und Musik / Abb. 17)^; ...

... und im Grün-Rot des Begehrens der toten Geliebten, zu der sich Judy verwandeln muss, um gesehen $\mathrm{zu}$ werden - sich verengend (1:39:53-1:41:04 / letzte Ausdrucksbewegungseinheit einer szenischen Komposition, Dominanten: Bildkomposition, Musik und Montage / Abb. 18)^. Die Redewendung „see a lot of each other" (Scotties Antwort auf Judys vorherige Frage, warum er für sie sorgen möchte) findet hier ihre negativierende Aktivierung, verkehrt sich doch die verbal aufgerufene Bildlichkeit im Zusammenspiel mit der Bildkomposition in die gegenteilige Wahrnehmungserfahrung - Judy verschwindet im Schwarz, ist nicht mehr als ein Schattenbild; ... 

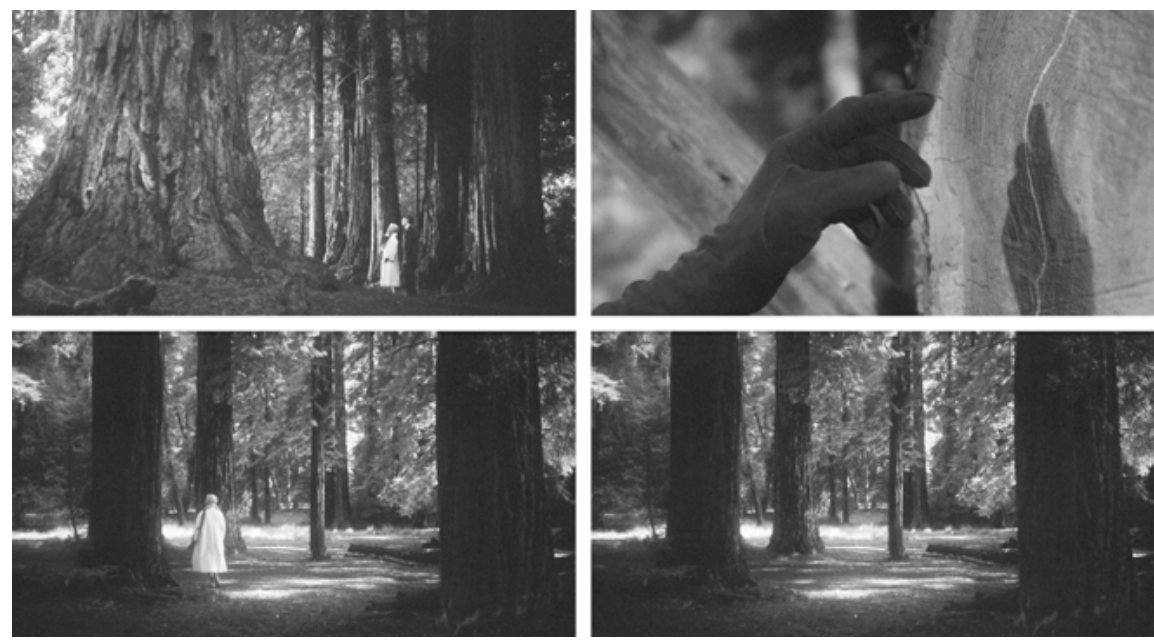

Abb. 17: VERTIGO. ( $\rightarrow$ Abb. 17*, Farbbogen)
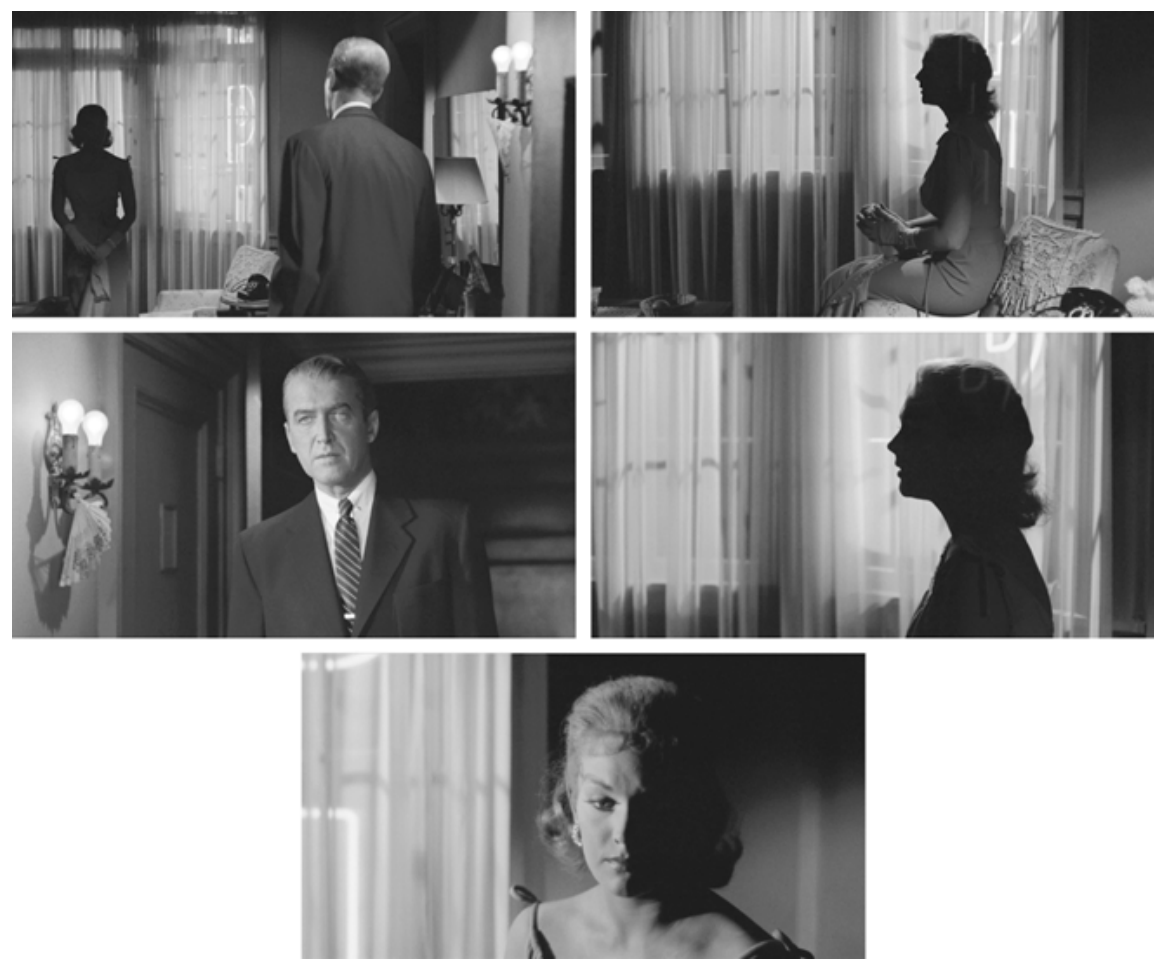

Abb. 18: vertigo. ( $\rightarrow$ Abb. 18*, Farbbogen) 
... bis das Begehren zuletzt ganz im Grün aufgeht, wenn in Judy die tote Madeleine wiederkehrt. Dieser Kulminationspunkt der so modellierten Denkbewegung eines in einem Komplementärverhältnis gründenden filmischen Metaphorisierens sei anhand zweier Ausdrucksbewegungen ausführlicher betrachtet - sind diese doch eben selbst ein Wiedergänger der Szene der ersten Begegnung, des ersten Sehens. Beschrieben werden die dritte (1:50:04-1:51:24) und vierte Ausdrucksbewegung (1:51:24-1:52:38) einer szenischen Komposition von vier Bewegungsmustern, die mit dem nervösen Warten von Scottie auf die in Madeleine verwandelte Judy in deren Hotelzimmer begonnen hatte.

Nach einem letzten schwachen Versuch des Widerstands fügt Judy sich dem Willen Scotties, mit gesenktem Kopf und noch offenem Haar wendet sie sich ab, die grüne Tür schließt sich hinter ihr. Das Bildfeld ist zweigeteilt: links das Rot, rechts das Grün.

Und dann, mit dem Einsatz eines leisen Moll-Streichertremolos im 4/4-Takt, mit sich immer wiederholenden Tonverläufen von unten nach oben und oben nach unten, hebt ein neues Bewegungsmuster an. Eine längere Einstellung zeigt Scottie, langsam zum Fenster gehend, dann ein kurzer Blick auf die geschlossene Tür. Scottie wendet seinen Blick der Tür zu - in der linksseitigen Ausrichtung spiegelverkehrt zu seiner Haltung, die er noch im indirekten Beobachten Madeleines an der Bar in Ernie's Restaurant eingenommen hatte. Das ganze Bild steht unter Spannung. Das Öffnen der Tür ist aus dem Off zu hören, die Lautstärke schwillt nach und nach an, im Tremolo steigen die Töne immer höher und höher - und zusammen mit einer schlagartig um ein Vielfaches erhöhten Schnittfrequenz wird das Tremolo durch ein kraftvolles, impulsives Legato abgelöst: Madeleine erscheint, geisterhaft schwach im Grünen zunächst, doch wird sie mit dem Fortgang der Schuss-Gegenschuss-Montage des direkten Anblickens immer weniger transparent - der Takt hat gewechselt, zu einem langsamen Walzer. ${ }^{9}$ Und erneut schraubt sich die Musik immer höher, ihr Tempo zieht an und dann, verbunden mit einer Tempodehnung, schreitet die Melodie in strahlenden, breiten Strichen herab. Wie in der Restaurant-Szene, nur ohne Verzögerung, bewegt sich Madeleine mit ihren Schritten auf Scottie zu, während zugleich die Kamera immer näher an Scottie heranrückt - bis beide Gesichter in Großaufnahme erscheinen. Das Grün ist raumgreifend. (Abb. 19)*

9 Angemerkt sei, dass diese beiden Szenen des erstmaligen Sehens mit unterschiedlichen musikalischen Motiven arbeiten. Zu den musikalischen Motiven in verTigo vgl. Schnellers Ausführungen hierzu (ebd.). 

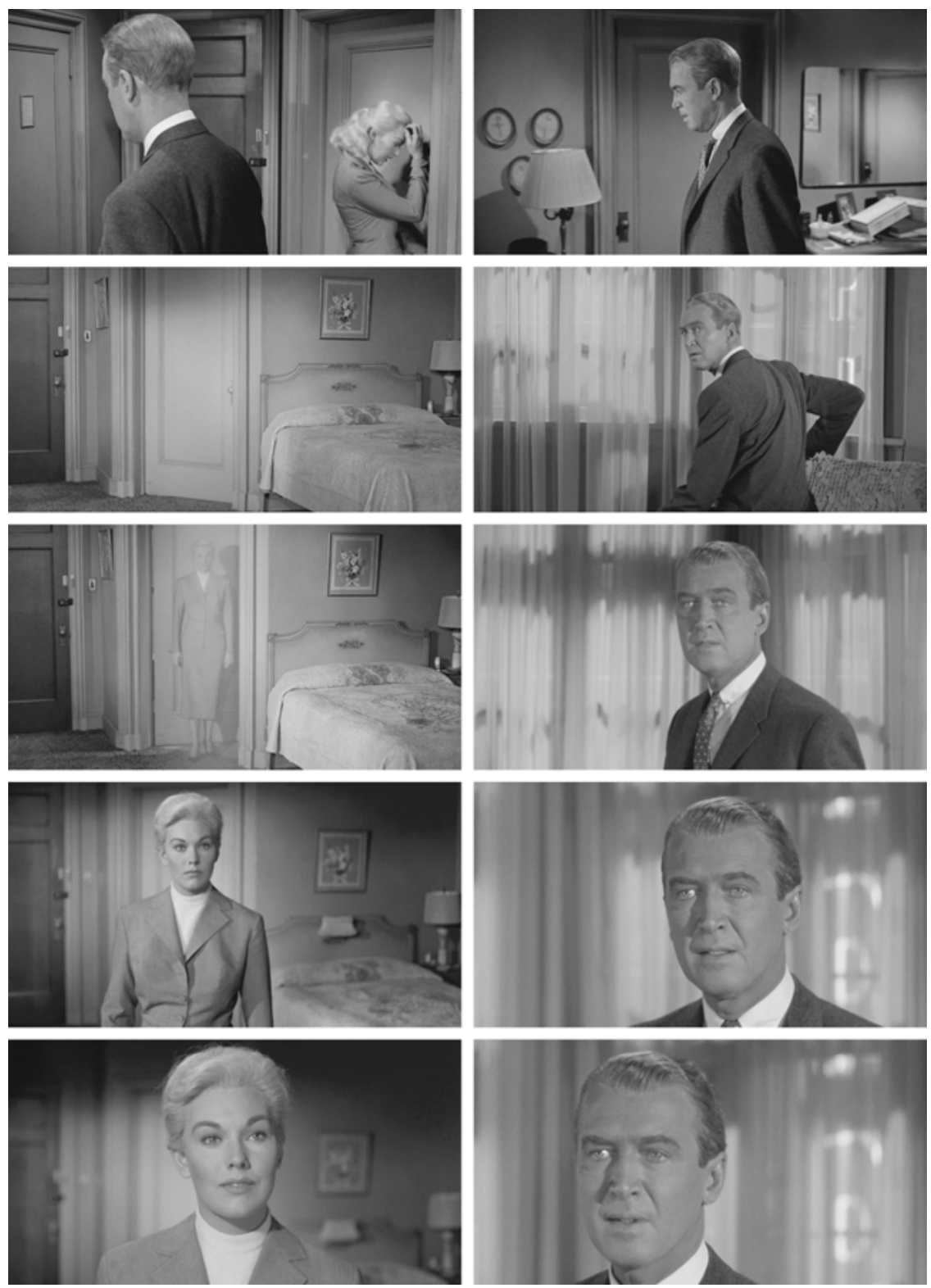

Abb. 19: Dritte Ausdrucksbewegung der Szene „Madeleine ein zweites Mal zum ersten Mal sehen“ - durch das Zusammenspiel von Montage und Musik entfaltet sich eine Annäherung und ein In-Erscheinung-Treten, deren Bewegungsqualität sich als die Transformation von Anspannung in anschwellende, sich entladende Kraft beschreiben lässt (VERTIGO, 1:50:041:51:25). ( $\rightarrow$ Abb. 19*, Farbbogen) 

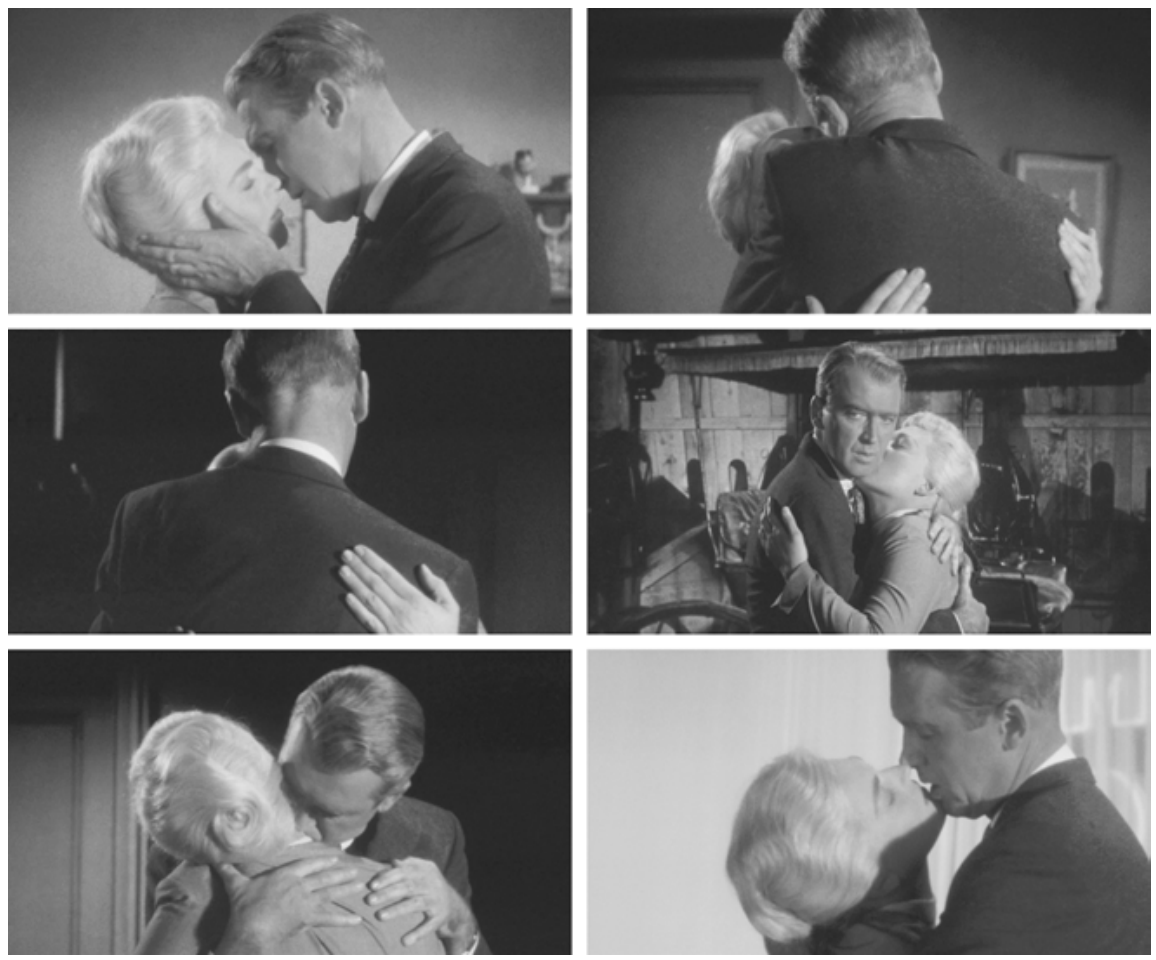

Abb. 20: Vierte Ausdrucksbewegung der Szene „Madeleine ein zweites Mal zum ersten Mal sehen“ - durch das Zusammenspiel von Kamerabewegung, Figurenbewegung und Musik wird das Erleben einer intensiven, nicht enden wollenden, sich in ihrer Intensität immer noch weiter steigernden Drehung evoziert (vertigo, 1:51:25-1:52:38). ( $\rightarrow$ Abb. 20*, Farbbogen)

Mit dem Erreichen der Großaufnahmen sind Scottie und Madeleine jetzt in einem gemeinsamen Bildfeld, und in einer einzigen langen Einstellung entfaltet sich eine nicht enden wollende Drehung (die Drehung des sich küssenden, haltenden Paares und das Umkreisen der Kamera desselben in gegenteiliger Richtung greifen ineinander) - es dehnt sich hier aus, was noch in der roten Komplementärszene am Filmanfang nach ein paar Sekunden schon wieder vorbei war. In dieser Ausdehnung verdunkelt sich der zuvor noch grau-grüne Umraum: Mit einem Flashback Scotties - sein Blick ist es, der schauend aus dem Kuss auftaucht - verändert sich die Szenerie in den Reitstall beim Kloster, wo Madeleine und Scottie sich küssten, bevor sie in den Tod stürzte. Das musikalische Tempo verdoppelt sich für einen Moment, bis dann musikalisch erneut in eine den Schluss- und Höhepunkt verzögernde Dehnung gegangen wird: Der Reitstall verschwindet, das Grün kommt wieder und Scottie taucht in das Küssen und Halten der Toten zurück - Madeleines Haar ist zu einer Spirale 
gedreht, und der breite, hohe Schlussakkord scheint endlos in seiner Kraft. (Abb. 20)*

Durch zahlreiche shiftings hat sich so das Emergieren eines dynamischen metaphorischen Wahrnehmungs-, Empfindungs- und Verstehensprozesses beschreiben lassen, ein Prozess, der ein Komplementärverhältnis von Liebessehnsucht und Todessehnsucht evoziert. ${ }^{10}$ Was die vertigo-Analyse exemplarisch zeigt: Ein filmisches Bild existiert nicht als Artefakt. Film meint vielmehr eine Figuration, ein Ereignis: ein immer schon doppelter, in sich verschränkter Wahrnehmungsvollzug, ein reflexives Wahrnehmungsverhalten. Film ist als ein verkörpertes, interaffektives, dynamisches Wahrnehmungsphänomen zu beschreiben, das sich erst im Akt des Sehens und Hörens, im Zuge der cinematic communication, im Filme-Sehen realisiert. Das Modell filmischen Metaphorisierens, das zu fassen sucht, wie in dieser Kommunikation Bedeutung entsteht, bedient sich dabei der geisteswissenschaftlichen Erklärungsfigur von System und Gebrauch, ohne jedoch ein dem Medienumgang genealogisch vorgelagertes System zu postulieren. Vielmehr, so die Überlegung, entsteht noch im Gebrauch selbst, im Diskursereignis eines Film-Sehens und -Hörens ein dynamisches, komplexes System, mit dem sich ein je eigenes, filmimmanent wirksames ,Zeichenregime' entfaltet.

Eine Möglichkeit, sich ein solches Diskursereignis und die damit verbundene situative, verkörperte Konstitution von Bedeutung rekonstruierend zu erschließen, ist, den Vollzug metaphorischer Aussagen zu beschreiben: Aussagen, die sinnliche und affektive Ähnlichkeiten und Bezüge zwischen Bereichen kreieren; Aussagen, die Welt und Wirklichkeit hervorbringen, und dies immer schon dynamisch, dialogisch, interaffektiv, in ihrer Verfasstheit als Bewegungsbilder realisieren. ${ }^{11}$ Metaphorische Mappings sind hier dann selbst noch maßgeblich die

10 Dieses evozierte Komplementärverhältnis von Liebes- und Todessehnsucht ist wiederum selbst noch Teil eines weiteren umfassenden Metaphorisierens im Ganzen, das an dieser Stelle nur vorläufig skizziert werden kann: VERTIGO elaboriert in vielfacher Weise ein Sehen - sei es in Gavins Aufforderung zu Beginn an Scottie, „Look [...]. You can see her there“, und der sich damit verknüpfenden Wahrnehmungserfahrung eines Begehrens, einer Liebe auf den ersten Blick; sei es gegen Ende durch den Ausspruch „see a lot of each other“, zusammen mit einem Entzug des Sehens; sei es durch Gemälde und Rahmungen; sei es in den endlosen Konstellationen des Beobachtens und Anblickens, die den gesamten Film durchziehen ... Und das Sehen ist von Beginn an ein Schwindel: das Auge der Frau, vom Vertigo-Schriftzug und von Spiralen durchdrungen; der Schwindel im Angesicht des Abgrunds ...: Liebe ist in der Unmöglichkeit eines schwindelfreien Sehens erfahrbar - und ist darin Tod und Trugbild zugleich.

11 Metaphorische Aussagen sind damit von narrativen Aussagen, von denen etwa bei Metz' Idee vom Film als einem Sprechen ohne Sprache die Rede ist, zu unterscheiden - mit einer Fokussierung auf narrative Aussagen stellt sich, wie Deleuze unterstreicht, das Problem, dass Film letztlich 
dynamisch sich entfaltenden Prozesse des Entstehens und Aufeinander-Bezogenseins von Erfahrungsbereichen - zentraler Referenzrahmen eines solchen „mapping in time“ ist entsprechend der jeweilige Film. ${ }^{12}$,Bedeutung' meint hier nichts Fixes, Gegebenes, Transportierbares, sondern etwas grundlegend Fluides und Flüchtiges, das in der Zeit, in und mit der dynamischen Materialität des Medienumgangs zur Existenz kommt, im Erleben des sich durch die filmische Expressivität, im filmischen Verhalten sich zeigenden Erlebens (im Ausdruck von Erfahrung durch Erfahrung). ${ }^{13}$,Bedeutung meint so letztlich selbst auch noch die zeitliche Verlaufsform des Metaphorisierens audiovisueller Diskurse, Verlaufsformen einer affektiven Bedeutungsmodellierung, bei der Form und Inhalt, Signifikat und Signifikant nicht mehr wirklich voneinander getrennt zu denken sind.

In einem solchen Sinne meint ,Metapher‘ ein Phänomen, das auf konkret sich ereignenden Wahrnehmungsprozessen beruht und einen Zusammenhang mit Bewegung hat; das sich als etwas Multimodales beschreiben lässt. Etwas, das keine präexistente Struktur ist, die sich eine Ausdrucksform sucht, sondern etwas Dynamisches, Prozesshaftes, das an eine konkrete Kommunikationssituation gebunden und dementsprechend komplex ist. Mit ,Metapher' sind konkret sich ereignende Prozesse des Fühlens und Erlebens angesprochen, die in einem verstehenden Begreifen münden. In diesem Sinn ist von ,Metapher hier als einem verkörperten Denken, als der Zuschaueraktivität des filmischen Metaphorisierens die Rede, vom audiovisuellen Bild modelliert und anhand dessen zu rekonstruieren. Vorgeschlagen ist mit dem filmischen Metaphorisieren ein Ansatz, mit dem sich über eine ästhetische Organisation von Wahrnehmung modellierten Semioseprozessen, einem Denken in Bildern genähert werden kann und dabei die miteinander verwobenen Ebenen von dynamischer Bildlichkeit berücksichtigt, mit denen audiovisuelle Bilder in ihrer gestischen wie sprachlichen Dimension mit ihren Zuschauern kommunizieren.

Der folgende dritte und letzte Teil der vorliegenden Arbeit vertieft dies nun noch einmal mit drei weiteren Analysen, die unterschiedliche Filmformen berücksichtigen. Mit den Rekonstruktionen, wie hier Prozesse emergieren und sich voll-

als Text präsentiert wird und infolgedessen „eine falsche Existenzweise“ verliehen bekommt, „insofern man ihm die Bewegung, das Charakteristischste seiner sichtbaren Eigenschaft, entzieht“. (Deleuze: Das Zeit-Bild, S. 42.)

12 Vgl. zum „mapping in time“ auch Schmitt/Greifenstein/Kappelhoff: Expressive Movement and Metaphoric Meaning, S. 2105-2107. Dies grenzt sich entsprechend vom Mapping-Verständnis $\mathrm{ab}$, wie es für die KMT wie auch für Forceville in Kapitel 1 der vorliegenden Arbeit erläutert wurde. 13 Für eine Kontextualisierung dessen in der breiteren theoretischen Diskussion zum Filmverstehen vgl. Kappelhoff/Greifenstein: Audiovisual Metaphors sowie Müller/Kappelhoff: Cinematic Metaphor und Kappelhoff: Kognition und Reflexion. 
ziehen, in denen ein Verstehen von etwas dadurch evoziert wird, dass etwas durch etwas anderes erfahren wird, soll auch die breitere medienwissenschaftliche Relevanz und Tragfähigkeit des Ansatzes des filmischen Metaphorisierens exemplarisch gezeigt werden. Mit Tom Tykwers märchenhaften Melodram DER KRIEGER UND DIE KAISERIN (D 2000) sei daher zunächst ein weiterer Spielfilm betrachtet. In der Folge wird sich dann mit einem ARD-Politmagazinbeitrag zur Bankenkrise (aus dem Jahr 2008) einem klassischen journalistischen Fernsehformat zugewendet. Und mit der abschließenden Analyse kehrt die Arbeit gewissermaßen zu ihrem Anfang zurück, wenn mit einem Hornbach-Spot (aus dem Jahr 2007) ein Werbefilm untersucht wird. Jeweils im Ganzen betrachtet, werden im Zuge dieser Analysen nicht zuletzt variierende dynamische Formen des Metaphorisierens, das heißt Verlaufsformen eines verkörperten Denkens in den Blick kommen, in denen auch die Weisen, wie Sprache darin Eingang findet, variiert. 